\title{
ECG Electrode Placements for Magnetohydrodynamic Voltage Suppression and improving Cardiac Gating in high-field MRI
}

\author{
Thomas S Gregory ${ }^{1 *}$, John Oshinski ${ }^{2}$, Ehud J Schmidt ${ }^{3}$, Zion T Tse ${ }^{1}$ \\ From 19th Annual SCMR Scientific Sessions \\ Los Angeles, CA, USA. 27-30 January 2016
}

\section{Background}

The accuracy of Electrocardiogram (ECG) gating for synchronization of MR scanner image acquisition and cardiac electrical activity is of great importance for acquiring high-quality Cardiac Magnetic Resonance (CMR) images free of motion artefacts. The distortion of ECG traces by Magnetohydrodynamic Voltages (VMHD) induced by interaction between the MRI static magnetic field $\left(\mathrm{B}_{0}\right)$ and rapid left-ventricular blood ejection during systole can lead to false and/or intermittent QRS complex detection and images with severe motion artefacts [1]. We hypothesized that an optimized electrode placement for the reduction of induced VMHD could be derived based on a thoracic model to increase the accuracy of QRS complex detection.

\section{Methods}

A vector model based on thoracic geometry [2] was calibrated using 12-lead ECGs recorded in four subjects in a GE 3T scanner to estimate VMHD distributions on the thorax. 4-lead ECG electrode placement was then optimized to: (1) minimize VMHD magnitude and (2) reduce displacement from the SA node for maximizing QRS complex amplitude (Figure 1a,b). A gradientdescent optimization routine was utilized to predict the optimal 4-lead ECG placement based on angular displacement and heart/aorta geometry (Figure 1c,d). Model results were then validated using five healthy subjects. Sensitivity $(\mathrm{Se})$ and Positive Predictability $(+\mathrm{P})$ rates for detection of R-waves were compared between conventional and MHD-suppressed lead placements for singlelead QRS complex detection [3].

${ }^{1}$ College of Engineering, University of Georgia, Athens, GA, USA

Full list of author information is available at the end of the article

\section{Results}

A $43.41 \%$ reduction in VMHD during the $\mathrm{S}$ - $\mathrm{T}$ segment (Figure 1f) was observed in ECGs using the MHD-suppressed placement relative to the conventional placement, while preserving the QRS complex (Figure 1e), resulting in an average increase in the $\mathrm{Se}$ and $+\mathrm{P}$ rate of $14.22 \%$ and $15.48 \%$, respectively (Figure $1 \mathrm{e}-\mathrm{g}$ ). $\mathrm{R}_{\text {peak }}$ amplitude inside the MRI in the MHD-suppressed placement had $<5 \%$ deviation from the standard placement outside of the MRI (Figure 1e). As compared to the conventional electrode placement (Figure 1c-d), MHD suppression may result from decreased visibility of the aorta through the lungs at the MHD-suppressed placement.

\section{Conclusions}

Electrode placement recommendations were computed and validated in a $3 \mathrm{~T}$ MRI, illustrating an increased accuracy in QRS complex detection using the MHDsuppressed placement.

\section{Authors' details \\ 'College of Engineering, University of Georgia, Athens, GA, USA. ${ }^{2}$ Radiology, Emory University Hospital, Atlanta, GA, USA. ${ }^{3}$ Radiology, Brigham and Women's Hospital, Boston, MA, USA.}

Published: 27 January 2016

\section{References}

1. Gregory: MRM 2014

2. Oostendorp: IEEE 2004.

3. Pan: IEEETransBiomed 1985.

doi:10.1186/1532-429X-18-S1-P328

Cite this article as: Gregory et al:: ECG Electrode Placements for

Magnetohydrodynamic Voltage Suppression and improving Cardiac Gating

in high-field MRI. Journal of Cardiovascular Magnetic Resonance 2016 18(Suppl 1): P328. 
a) Thoracic model to predict minimum and maximum VMHD in electrodes based on angular displacement between the electrode vector and the VMHD vector. Electrode angular displacement (red line) and Electrode VMHD distribution (blue line) are shown. Electrode VMHD distribution (blue line) was calculated using Inverse Correlation Coefficients between 12lead ECGs obtained inside and outside of the bore, whereas a 0 represents no MHD voltage while a 1 represents full MHD voltage. Electrode angular displacement (red line) is the angle between the electrode vector and lead I (frontal plane).

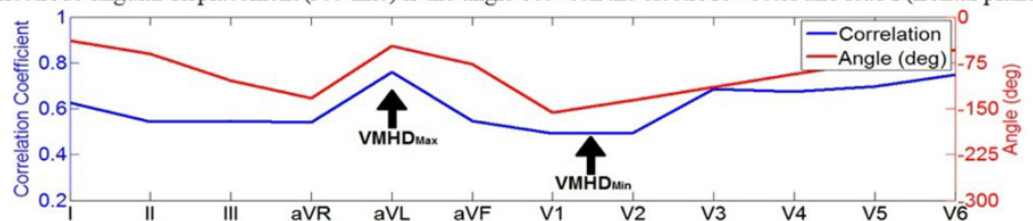

b) Mapped electrical vectors (green) from 12-lead ECG in the thoracic model [1], used to determine angular displacement between leads and VMHD during model validation.

c) Standard electrode placement for ECG gating (red). Electrical vector from electrodes is nearly unobscured from heart and aorta by the lungs.

d) MHD-suppressed electrode placement (blue). Electrical vector is in view of heart, but remains obscured from aorta by superior lobe of the lung.

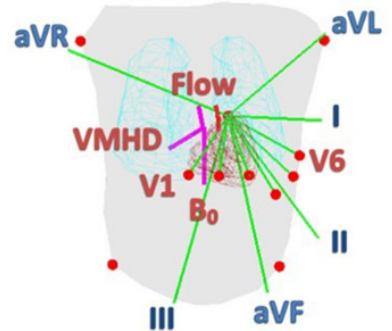

e) 4-lead ECG recordings obtained outside the MRI in the standard and MHD-suppressed locations. R Reak in the MHD-suppressed case was found to have a $<5 \%$ deviation from the standard placement.

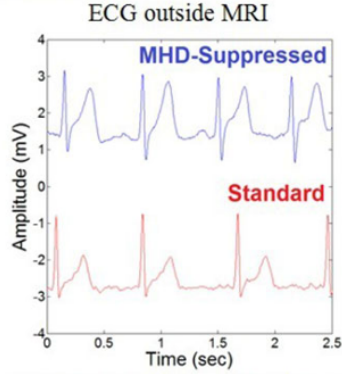

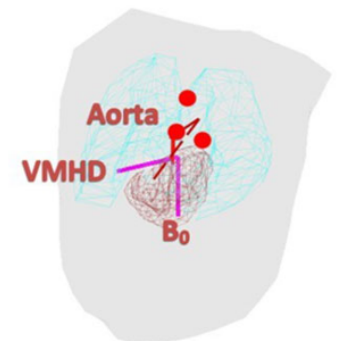

f) ECG recordings obtained inside the MRI in the standard and MHDsuppressed locations. VMHD was reduced by $59 \%$ in the MHDsuppressed case relative to the standard placement.

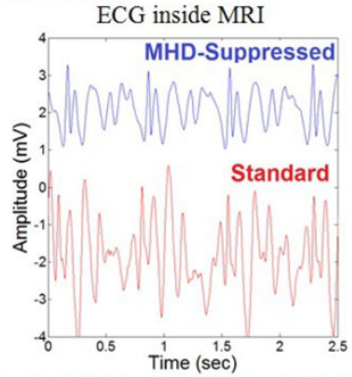

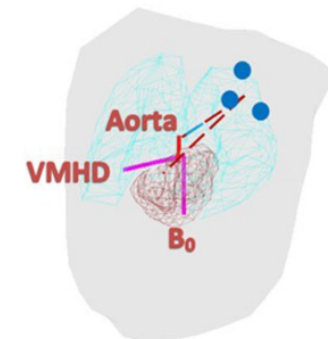

g) Subject transverse slice of ascending and descending aorta acquired using ECG-gating.

Table 1: Evaluation of MHD suppression in Each Subject for QRS complex Detection

\begin{tabular}{|c|c|c|c|c|c|}
\hline & \multicolumn{2}{|c|}{$\begin{array}{l}\text { Improvement in } \\
\text { ORS Complex Detection }\end{array}$} & \multicolumn{3}{|c|}{$\begin{array}{c}\text { Level of MHD Suppression in ECG Segments } \\
\text { (Peak-to-Peak) }\end{array}$} \\
\hline & $\mathrm{Se}$ & $+P$ & $P$-wave & QRS Complex & $S-T$ segment \\
\hline $\begin{array}{c}\text { Subject } 1 \\
M / 20 \text { yrs } / 108 \mathrm{~kg}\end{array}$ & $3.10 \%$ & $14.59 \%$ & $0.44 \mathrm{mV}(25.41 \%)$ & $0.27 \mathrm{mV}(11.32 \%)$ & $2.55 \mathrm{mV}(64.38 \%)$ \\
\hline $\begin{array}{c}\text { Subject } 2 \\
M / 20 y r s / 72 \mathrm{~kg}\end{array}$ & $8.33 \%$ & $26.67 \%$ & $0.06 \mathrm{mV}(23.19 \%)$ & $0.14 \mathrm{mV}(17.25 \%)$ & $0.87 \mathrm{mV}(55.39 \%)$ \\
\hline $\begin{array}{c}\text { Subject } 3 \\
M / 21 \text { yrs } / 68 \mathrm{~kg}\end{array}$ & $25.00 \%$ & $33.33 \%$ & $0.13 \mathrm{mV}(18.50 \%)$ & $0.36 \mathrm{mV}(38.91 \%)$ & $0.52 \mathrm{mV}(28.21 \%)$ \\
\hline $\begin{array}{c}\text { Subject } 4 \\
M / 27 y r s / 82 \mathrm{~kg}\end{array}$ & $11.11 \%$ & $11.11 \%$ & $0.09 \mathrm{mV}(33.72 \%)$ & $0.18 \mathrm{mV}(44.61 \%)$ & $0.10 \mathrm{mV}(19.27 \%)$ \\
\hline $\begin{array}{c}\text { Subject } 5 \\
M / 26 y r s / 77 \mathrm{~kg}\end{array}$ & $23.53 \%$ & $13.93 \%$ & $0.33 \mathrm{mV}(52.64 \%)$ & $0.07 \mathrm{mV}(10.36 \%)$ & $0.89 \mathrm{mV}(49.89 \%)$ \\
\hline Mean & $14.22 \%$ & $15.48 \%$ & $0.21 \mathrm{mV}(30.69 \%)$ & $0.20 \mathrm{mV}(24.49 \%)$ & $0.99 \mathrm{mV}(43.41 \%)$ \\
\hline Std. Dev. & $9.63 \%$ & $16.99 \%$ & $0.16 \mathrm{mV}(13.45 \%)$ & $0.12 \mathrm{mV}(16.11 \%)$ & $0.93 \mathrm{mV}(18.96 \%)$ \\
\hline
\end{tabular}

